\title{
Temporal Pattern of Borrelia burgdorferi p21 Expression in Ticks and the Mammalian Host
}

\author{
Subrata Das, ${ }^{\star}$ Stephen W. Barthold,, Susan Stocker Giles, ${ }^{\star}$ Ruth R. Montgomery, ${ }^{\star}$ Sam R. Telford III, ${ }^{\S}$ and Erol Fikrig* \\ *Section of Rheumatology, Department of Internal Medicine, Yale University School of Medicine, New Haven, Connecticut 06520-8031; \\ ${ }^{\ddagger}$ Section of Comparative Medicine, Yale University School of Medicine, New Haven, Connecticut 06520-8031; ${ }^{\S}$ Department of Tropical \\ Public Health, Harvard University School of Public Health, Boston, Massachusetts 02115
}

\begin{abstract}
The temporal synthesis of the P21 protein of Borrelia burgdorferi and the development of the humoral response to this antigen was assessed in infected mice. $p 21$ is a member of the 0 spE-F gene family and its protein, $\mathrm{P} 21$, has been shown to be expressed by $B$. burgdorferi within infected mice but not by spirochetes cultured in vitro. P21 was not detected on $B$. burgdorferi in unfed or engorged Ixodes dammini (also known as I. scapularis) ticks, further supporting the postulate that P21 synthesis is specific for the mammalian host. In $B$. burgdorferi-infected mice, $o s p E \mathrm{mRNA}$ and OspE antibodies were observed at $7 \mathrm{~d}$, whereas $p 21 \mathrm{mRNA}$ and P21specific antibodies were detected at 21-28 d, suggesting that p21 is expressed later than ospE. Moreover, ospA mRNA was not discernible until day 14, indicating that $\operatorname{osp} A$, like p21, is not expressed in the early stages of tick-transmitted murine Lyme borreliosis. Because $p 21$ is expressed during infection in mice, we assessed the human humoral response to $\mathrm{P} 21.28 \%$ (34 of 122) of the patients with either early- or late-stage Lyme disease, and 33\% (11 of 33) of the individuals with Lyme arthritis had P21 antibodies, suggesting that a P21 response may serve, at least partially, as a marker of infection. Active immunization with recombinant P21 did not protect $\mathrm{C} 3 \mathrm{H}$ mice from tick-borne $B$. burgdorferi infection, and passive transfer of $\mathrm{P} 21$ antiserum to infected mice did not alter the course of disease. These data suggest that the antigenic structure of $B$. burgdorferi changes during the early stages of murine infection. (J. Clin. Invest. 1997. 99: 987-995.) Key words: Lyme disease • spirochete • arthritis • ospE $\bullet$ ticks
\end{abstract}

\section{Introduction}

Characterization of the immune response to Borrelia burgdorferi antigens that are synthesized during the spirochete's lifecycle can yield information on the extent to which this organism

Address correspondence to Erol Fikrig, M.D., Section of Rheumatology, Department of Internal Medicine, Yale University School of Medicine, 610 Laboratory of Clinical Investigation, 333 Cedar Street, New Haven, CT 06520-8031. Phone: 203-737-4080; FAX: 203-7857053; E-mail: erol_fikrig@qm.yale.edu

Received for publication 9 July 1996 and accepted in revised form 9 December 1996.

J. Clin. Invest.

(C) The American Society for Clinical Investigation, Inc. 0021-9738/97/03/0987/09 \$2.00

Volume 99, Number 5, March 1997, 987-995 alters its antigenic structure. B. burgdorferi gene expression can vary both in vivo and in vitro, and $b b k 2.10$, epp $A, p G$, and $p 21$ (the subject of this study) may be induced selectively during infection (1-3). To specifically identify immunogenic $B$. burgdorferi genes selectively expressed in vivo, our group devised a differential screening strategy in which a $B$. burgdorferi expression library was probed with sera from $B$. burgdorferiinfected mice (immune sera), compared to hyperimmune sera against heat-killed spirochetes: clones that reacted with immune, but not hyperimmune, sera reflected antigens likely to be expressed selectively in vivo (4). Using this technique, we identified $p 21$, and five other genes (or gene families) that are expressed by $B$. burgdorferi within infected mice but not by spirochetes cultured in Barbour-Stoenner-Kelly (BSK) ${ }^{1}$ II medium at $33^{\circ} \mathrm{C}$. $\mathrm{P} 21$ has $71 \%$ homology with outer surface protein (Osp) E, one of the gene-products of bicistronic $o s p E-F$ operon that is expressed by cultured spirochetes (5). Recent reports of two homologues to $O s p E$, designated $\operatorname{erp} A$ and $\operatorname{erp} C$, and five homologues of $\operatorname{sp} F$, designated $\operatorname{erp} B, \operatorname{erp} D$, $p G, b b k 2.10$, and $b b k 2.11$, suggest that $\operatorname{osp} E-F, p 21, \operatorname{erp} A-B$, $\operatorname{erp} C-D, p G, b b k 2.10$, and $b b k 2.11$ are part of a single gene family in the $B$. burgdorferi genome $(1,3-6)$.

Two of the major B. burgdorferi antigens, OspA and OspC, are differentially expressed within Ixodes dammini (also known as I. scapularis) ticks. OspA is abundant on spirochetes within the midguts of unfed ticks and is sparse or absent on B. burgdorferi within feeding ticks (7). In contrast, OspC is not present on $B$. burgdorferi in flat ticks, but is readily detected on spirochetes within fed ticks (8). Moreover, studies assessing OspA and OspC expression in mice inoculated with $10^{4} \mathrm{~B}$. burgdorferi (by syringe-challenge) revealed that OspC is expressed early in infection, while OspA can be detected at 14, but not $30 \mathrm{~d}$ after challenge (9). The lack of OspA expression during the early stages of murine infection may account, at least in part, for the absence of a prominent OspA antibody response early in infection, even though OspA is one of the most plentiful proteins on spirochetes cultured in BSK II medium. At some time following infection, however, OspA must be synthesized because some patients with Lyme disease, and mice challenged with low doses $\left(10^{2}\right)$ of spirochetes, develop OspA antibodies during infection (10-12). Collectively, these studies show that diverse $B$. burgdorferi proteins are expressed in various stages of the spirochete-tick life cycle and different stages of infection in the mammalian host. As B. burgdorferi can alter its structure, and the $o s p E-F$ gene family may be selectively ex-

1. Abbreviations used in this paper: aa, amino acid; BSK, BarbourStoenner-Kelly; GT, glutathione S-transferase; RT, reverse transcriptase. 
pressed, we have now performed the first studies that follow the temporal expression of an induced gene, in this case, $p 21$, as spirochetes migrate from the tick to the mammalian host, and then during the evolution of murine infection.

\section{Methods}

Pulsed-field gel electrophoresis. Pulsed-field gel electrophoresis was performed in a CHEF-DR ${ }^{\circledR}$ III (Bio-Rad Laboratories, Richmond, CA) pulsed-field gel electrophoresis system using B. burgdorferi N40 DNA (13). DNA plugs containing $10^{8} B$. burgdorferi were treated with sarkosyl, lysed overnight with proteinase $\mathrm{K}$ and then loaded onto a $0.8 \%$ agarose gel. The electrophoresis was done at $14^{\circ} \mathrm{C}$ for $18 \mathrm{~h}$ at $198 \mathrm{~V}$ in Tris-borate-EDTA buffer, with a ramped pulse time of 1-30 s. For two-dimensional electrophoresis, the gel was again electrophoresed after changing the direction at 90 degrees at $80 \mathrm{~V}$ for $6 \mathrm{~h}$. The DNA was transferred to a Hybond $\mathrm{N}^{+}$nylon membrane and incubated with a $p 21$ (nucleotides 55-561 of the $p 21$ gene), osp $A$, ospE (nucleotides 49-249 of the $o s p E$ gene), flagellin, or $p 30$ probe at $55^{\circ} \mathrm{C}$ for $14 \mathrm{~h}$. The membrane was then washed three times $(0.5 \times \mathrm{SSC})$. The binding of the $p 21$ probe to three specific DNA bands was visualized in the Southern blot by the enhanced chemiluminescence (ECL) method (Amersham Corp., Arlington Heights, IL). A p21-specific oligonucleotide probe (TAAATCTTTACTAGTTGCATAATTCTTGCA) was hybridized to the electrophoresed B. burgdorferi DNA to specifically localize $p 21$. Appropriate controls localized the $950 \mathrm{~kb}$ linear chromosome ( $p 30$, a substrate-binding protein homologue [14] that we have previously localized to the chromosome in $B$. burgdorferi $\mathrm{N} 40$, and flagellin) and the $49 \mathrm{~kb}$ plasmid (ospA).

Expression and purification of recombinant $P 21$ and generation of $P 21$ antiserum. To express and purify P21, the sequence of $p 21$ lacking the hydrophobic leader region (nucleotides 1-54) was amplified by PCR. BamHI (5') and HindIII (3') restriction endonuclease digestion sites were added to the primers $\left(5^{\prime}\right.$-AGTCGGATCCAAGATTCATACTTTATCGATG- $3^{\prime}$ and $5^{\prime}$-AGTCAAGCTTCTATTTTAAATTTTTTTTGATC- $3^{\prime}$ ) to facilitate cloning of $p 21$ into the $p M X$ vector (15). $50 \mathrm{ng}$ of plasmid, excised by the R408 helper phage, was used as the template for PCR. PCR was performed for 30 cycles, with a denaturation temperature of $94^{\circ} \mathrm{C}$ for $1 \mathrm{~min}$, annealing temperature of $55^{\circ} \mathrm{C}$ for $1 \mathrm{~min}$, and extension temperature of $72^{\circ} \mathrm{C}$ for $1 \mathrm{~min}$. $p 21$ was digested with BamHI and HindIII, and cloned in frame with the glutathione $S$-transferase $(G T)$ gene in the $p M X$ vector. The new vector was designated $p M X-p 21$. The GT-P21 recombinant fusion protein was synthesized in Escherichia coli $\mathrm{DH} 5 \alpha$ and the antigen was purified from the whole-cell-lysate using a glutathione-sepharose-4B column (Pharmacia Biotech, Uppsala, Sweden).

3 -wk-old female $\mathrm{C} 3 \mathrm{H} / \mathrm{HeN}$ mice were immunized with $10 \mu \mathrm{g}$ of GT-P21 fusion protein or the 10 amino acid (aa) P21-specific peptide (NNELKVKQSN) bound to BSA in complete Freund's adjuvant, and boosted with $10 \mu \mathrm{g}$ of antigens in incomplete Freund's adjuvant at 15 and $25 \mathrm{~d}$ (16). The aa sequence comparison between P21 and OspE revealed a 10 consecutive aa sequence (position 36-45) in P21 that did not have homology with any region of OspE (4). This sequence was biochemically synthesized and bound to BSA. Control mice were immunized with purified GT or BSA in an identical fashion. Sera were obtained from the animals $15 \mathrm{~d}$ after the final boost.

Human and murine immune sera. Patient's sera were obtained from the Yale Lyme Disease Clinic and the Centers for Disease Control (CDC). Individuals were classified as having early- or late-stage Lyme disease based on the clinical presentation as documented by a physician, and serum antibodies to B. burgdorferi, according to CDCdefined disease criteria. $78 \%$ of the patients that donated samples to the CDC were culture-positive for B. burgdorferi. Patients from the Yale clinic were not assessed for infection by culture. To obtain sera from infected mice, five animals were intradermally inoculated with $10^{4}$ cultured $B$. burgdorferi and serum samples were obtained 7, 14, $28,60,90$, and $180 \mathrm{~d}$ after the challenge.
Immunoblot and ELISA. To determine the presence of P21 antibodies in the sera of patients with Lyme disease or in infected mice, BSA-linked P21-specific peptide or GT-P21 $(0.5 \mu \mathrm{g} / \mathrm{dot})$ was dotted on nitrocellulose membranes according to our published protocols (4). BSA or GT were used as controls. Nitrocellulose strips were then incubated with the sera (1:100 dilution) for $4 \mathrm{~h}$ and alkaline phosphatase conjugated goat-anti-human or goat-anti-mouse antibody (IgM or $\mathrm{IgG}$ ). Blots were developed for $5 \mathrm{~min}$ in nitroblue tetrazolium and 5-bromo-4-chloro-3-indolyl phosphate (NBT-BCIP) (Stratagene, Inc., La Jolla, CA), and the reaction was quenched in distilled $\mathrm{H}_{2} \mathrm{O}$.

For ELISA, 96-well Titertek microtitration plates (ICN Biomedicals, Inc., Irvine, CA) were coated with BSA, BSA-linked P21-specific peptide, GT, GT-P21, or GT-OspE $(1 \mu \mathrm{g} / \mathrm{ml}$ of carbonate coating buffer, $100 \mu \mathrm{l} /$ well) at $4^{\circ} \mathrm{C}$ for $18 \mathrm{~h}$ (17). To prevent non-specific binding of antibodies, blocking was performed with $100 \mu$ l of $10 \%$ FCS in PBS for $1 \mathrm{~h}$. Wells were then washed three times with PBS containing $0.1 \%$ Tween-20 (PBST). Human or mouse sera, diluted 1:100, were added to the wells in triplicates, incubated for $8 \mathrm{~h}$ at $4^{\circ} \mathrm{C}$, and then washed three times with PBST. Goat anti-human or antimouse IgM or IgG coupled to alkaline phosphatase (Sigma Chemical Co., St. Louis, MO) at a dilution of 1:2000 and was used to detect the primary antibodies. The substrate for alkaline phosphatase was $p$-nitrophenol phosphate at a concentration of $1 \mathrm{mg} / \mathrm{ml}$ in glycine buffer (Sigma Chemical Co.).

RT-PCR of flagellin, ospA, p21, and ospE from infected mice. Mice were engorged upon by 15 B. burgdorferi N40-infected ticks, and then killed on days 7, 14, 21, 28, 60, and 120 after tick feeding (7). Total RNA, from 3-4 mice for each time point, was isolated from murine spleens using the Micro RNA isolation kit (Stratagene, Inc.) (4). First strand cDNA was synthesized with random primers from $5 \mu \mathrm{g}$ of total spleen RNA from individual infected mouse. A control experiment containing no reverse transcriptase (RT) was also performed for each sample to check for possible DNA contamination. Four sets of specific upstream and downstream primers were used to amplify: flagellin (5'-ATTAATGCTCAAATAAGAGG-3', 5' -CCGCCTTGAGAAGGTGCTGT-3'), osp $A$ (5'-TCTGACGATCTAGGTCAAACCACA-3' ${ }^{\prime}, 5^{\prime}$-TTTAGTTTTTTTACTGTTTACAGTAATTGT-3'), p21 (5' -AATAATGAGTTAAAAGTTAAGCAA- ${ }^{\prime}, 5^{\prime}$-TTCTAACTTCTATTTTAAATT-3'), and $o s p E$ (5'-ATAGGTGCTTGCAAGATTCAT-3', 5'-GCCGAATGTCCCCCAGCGTTTAAA-3') at denaturing, annealing, and extension temperatures of $94^{\circ} \mathrm{C}, 55^{\circ} \mathrm{C}$, and $72^{\circ} \mathrm{C}$, respectively, for $1 \mathrm{~min}$ at each step for 30 cycles.

Immunization studies. $15 \mathrm{I}$. dammini nymphs infected with $B$. burgdorferi $\mathrm{N} 40$ were fed to repletion on each of six mice immunized with GT-P21 or GT (control). Engorged ticks fell from the mice within $4 \mathrm{~d}$ and were collected in water baths below the cages. $2 \mathrm{wk}$ later, all mice were killed and examined for infection. Blood, bladder, spleen, and skin from the inoculation site and ear were cultured in BSK II medium (16). The tibiotarsi and the heart of each animal were microscopically evaluated for inflammation. Engorged ticks were examined for spirochetes on the 10th day after feeding. Individual ticks were lightly homogenized in $100 \mu$ l of PBS in a $1.5-\mathrm{ml}$ microfuge tube, an aliquot of $10-\mu l$ of the homogenate was spotted on a clean slide, air dried, and then fixed in cold acetone for $10 \mathrm{~min}$. The slides were incubated with fluorescein-isothiocyanate (FITC)-labeled rabbit-B. burgdorferi $\mathrm{N} 40$ antiserum, mounted under a coverslip, and examined on a Zeiss Axioskop fluorescence microscope.

To assess the effects of P21 antiserum on existing infection and disease, three groups of C.B 17-scid (SCID) mice (five animals in each group) were inoculated with $10^{4} \mathrm{~B}$. burgdorferi N40, then injected with $0.5 \mathrm{ml}$ of either GT-hyperimmune mouse serum, GT-P21hyperimmune mouse serum, or $90 \mathrm{~d}$ infected mouse serum (all diluted 1:10) 4, 8, and $12 \mathrm{~d}$ after inoculation (18). The mice were killed on day 15 and blood, bladder, spleen, and skin from the inoculation site, and the ear were cultured in BSK II medium. The tibiotarsi and heart of each mouse were examined microscopically for disease as previously described (16). 
Immunofluorescence. Groups of B. burgdorferi N40-infected I. dammini nymphs, including unfed ticks and ticks fed to repletion on $\mathrm{C} 3 \mathrm{H}$ mice, were checked for the presence of $\mathrm{P} 21$ on spirochetes within the vector by indirect immunofluorescence (7). Each tick was lightly homogenized in $100 \mu \mathrm{l}$ of PBS and $10-\mu l$ aliquots were spotted on a silylated glass slide. The slides were air-dried and then fixed with $4 \%$ paraformaldehyde and saponin. The specimens were incubated in P21-peptide-specific or OspE antisera (1:10 dilution) for $1 \mathrm{~h}$, washed, followed by incubation in anti-mouse or anti-rabbit IgG coupled with FITC (1:500 dilution) for another $\mathrm{h}$, and viewed under a Zeiss Axioskop fluorescence microscope. OspA monoclonal antibody CIII.78, which recognizes $B$. burgdorferi within unfed ticks, but does not readily detect spirochetes within fed ticks, was used as a positive control $(7,15)$. Flagellin monoclonal antibody $\mathrm{H} 9724$ was used to recognize $B$. burgdorferi from either of these two sources. BSA antiserum was used as the negative control.

Expression of $\mathrm{P} 21$ by spirochetes in the mice was detected by immunofluorescence of $B$. burgdorferi collected from the peritoneal lavage fluid of 21-day-old B. burgdorferi-infected mice (9). The peritoneal cavity of each of 10 mice was lavaged with $5 \mathrm{ml}$ of $\mathrm{Ca}^{++}$- and $\mathrm{Mg}^{++}$-free PBS and the fluid was collected. The cells and bacteria were pelleted at $10,000 \mathrm{rpm}$ for $3 \mathrm{~min}$, resuspended with PBS, fixed with $-20^{\circ} \mathrm{C}$ methanol, and then incubated with rabbit anti-B. burgdorferi N40 serum (1:1000 dilution) and either anti-BSA or anti-BSAP21-peptide sera (1:50 dilution) for $1 \mathrm{~h}$ to double-label each bacteria. FITC-conjugated goat anti-mouse IgG (1:200 dilution) and rhodamine-conjugated goat anti-rabbit $B$. burgdorferi IgG (1:200 dilution) were used as the secondary antibodies to visualize the spirochetes.

\section{Results}

$P 21$ is located on a circular plasmid. To localize $p 21$ in the $B$. burgdorferi genome, a 221 DNA fragment (nucleotide 55-561) was used to probe $B$. burgdorferi N40 DNA separated by pulsed-field gel electrophoresis (Fig. $1 a$ ). As expected, the $p 30$ and flagellin probes identified the chromosome (Fig. $1 a$, lane 1 [p30] and lane 5 [flagellin]) and the ospA probe hybridized with the $49 \mathrm{~kb}$ linear plasmid (Fig. $1 a$, lane 2). An $o s p E$-specific (201 bp) probe identified a single plasmid (Fig. $1 a$, lane 6 ). The full-length $p 21$ probe bound at three locations (Fig. $1 a$, lane 3 ), but the $p 21$-specific oligonucleotide probe recognized a single plasmid as the location of $p 21$ (Fig. $1 a$, lane 4) which was different from the $45 \mathrm{~kb}$ plasmid that contained the $o s p E$ gene (Fig. $1 a$, lane 6 ). To determine whether the plasmid that contained $p 21$ was circular, B. burgdorferi DNA was separated in two-dimensions, first by pulsed-field and then by constant voltage gel electrophoresis. The linear and circular DNA of $B$. burgdorferi, separated by pulsed-field electrophoresis in the first-dimension, migrated with different characteristics when run at a uniform and constant voltage in the second-dimension. Linear DNA migrate at a similar rate, whereas the circular DNA move more slowly (19). As expected, the linear DNA, including the chromosome ( 330 probe) and the $49 \mathrm{~kb}$ linear plasmid (ospA probe), migrated to a similar distance from the origin, whereas the plasmid containing $p 21$ moved to a different location suggesting that the $p 21$ plasmid is circular (Fig. $1 b)$.

P21 is expressed on spirochetes within infected mice, but not within infected ticks. To determine whether P21 was expressed by spirochetes in I. dammini, lysates of unfed and engorged ticks containing B. burgdorferi were probed with a P21-specific antiserum. Both types of ticks were examined, as B. burgdorferi gene expression varies in the unfed or fed vectors $(7,8)$.
Spirochetes were readily detected by flagellin-specific monoclonal antibody $\mathrm{H} 9724$ in both unfed and fed ticks (Fig. $2 a, A$ and $B$ ), and by OspA-monoclonal antibody CIII.78 in unfed, but not in engorged ticks (Fig. $2 a, C$ and $D$ ), consistent with

\section{a}

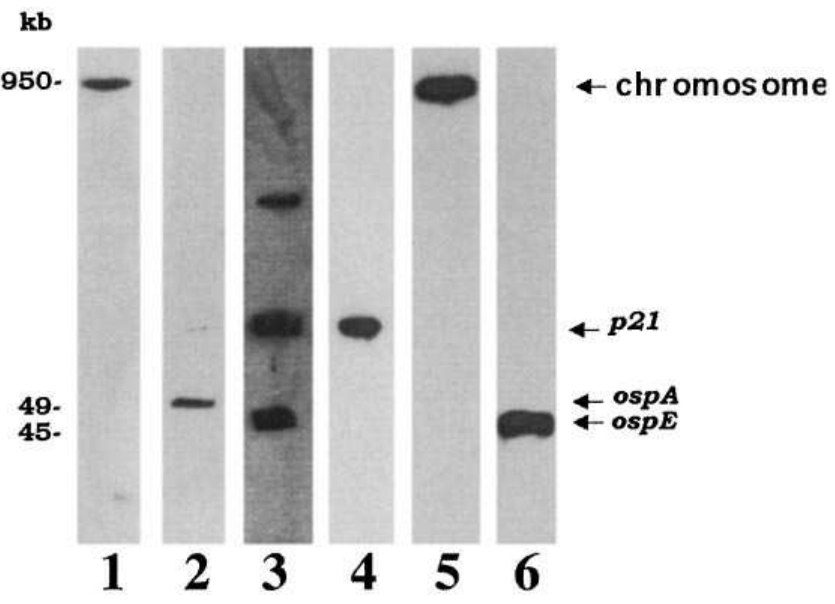

b

\section{Origin}

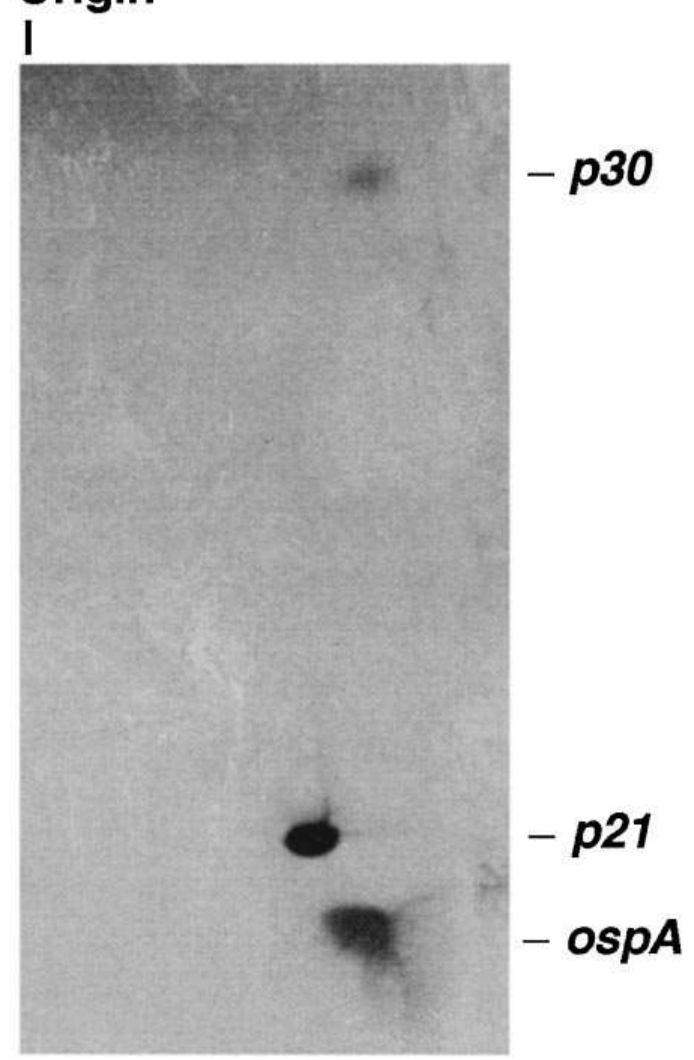

Figure 1. (a) Pulsed-field gel electrophoresis of B. burgdorferi DNA probed with $P 30$ (lane 1), ospA (lane 2), full-length $p 21$ (lane 3), a $P 21$-specific oligonucleotide (lane 4), flagellin (lane 5), and a 210-bp $o s p E$ fragment (lane 6) probes. (b) Two-dimensional gel electrophoresis (pulsed-field in dimension one and then constant voltage in dimension two of B. burgdorferi DNA. 
previous studies (7). OspE antibody also stained B. burgdorferi from both unfed and engorged ticks (Fig. $2 a, G$ and $H$ ). P21-specific immunofluorescence was not observed on $B$. burgdorferi within either the unfed or fed ticks (Fig. $2 a, E$ and $F)$. In contrast, $\mathrm{P} 21$-specific immunofluorescence was found to be present on the spirochetes isolated from infected mice (Fig. $2 b, B, D$, and $F)$. None of the spirochetes collected from peritoneal lavage fluid of 21 day-infected mice showed immunofluorescence when incubated with BSA antiserum (control) (Fig. $2 \mathrm{~b}, H)$. To insure that $\mathrm{P} 21$-specific antiserum selectively identifies P21 and not OspE, B. burgdorferi lysates, recombinant P21 and OspE were probed with OspE, P21 and P21-peptidespecific antisera. As expected, P21-specific antiserum readily recognized recombinant $\mathrm{P} 21$ but not OspE, indicating that the antiserum contained high titers of P21-specific antibodies (Fig. 2 c). The P21-specific peptide was also probed with P21 or OspE antisera to determine the specificity of these antisera. The P21-specific peptide was identified by antibodies in the P21 antiserum and not by OspE antiserum (Fig. $2 d$ ). Moreover, both anti-GT-P21 and anti-GT-OspE sera identified a single $19 \mathrm{kD}$ band (OspE) in B. burgdorferi lysates, whereas P21-peptide specific antiserum was unable to detect any antigen (Fig. $2 e$ ).

P21-specific antibodies appear after OspE antibodies in B. burgdorferi-infected mice. Recombinant GT-P21, GT-OspE, and P21-specific peptide were used as substrates in ELISA to determine the temporal development of P21 and OspE antibodies during murine infection. IgG antibodies to OspE and P21 were first detected $7 \mathrm{~d}$ after infection and persisted until $180 \mathrm{~d}$, the final time point in our studies (Fig. 3). As ospE and p21 share significant homology and OspE and P21 antibodies can cross react, it could not be determined whether the humoral responses were directed towards P21, OspE, or both. Therefore, an ELISA using the P21-specific peptide was used to detect P21-specific antibodies. P21-specific antibodies appeared in the sera of mice on day 28 and persisted throughout the course of infection.

p21 mRNA is detected at a later time point than ospE $m R N A$ in B. burgdorferi-infected mice. Because the P21-specific antibody response appears after the OspE response, we studied the genesis of $p 21$ and $o s p E$ mRNA in B. burgdorferiinfected mice. For this purpose, specific primers for $p 21$ and $o s p E$ were generated that do not amplify $o s p E$ or $p 21$, respectively (Fig. 4). These primer sets for $o s p E$ and $p 21$ amplified only a single band from $B$. burgdorferi N40 DNA, and sequence analysis confirmed that these DNA fragments were $o s p E$ and $p 21$, respectively. Thus, the primers for $p 21$ and $o s p E$ are both specific. We also assessed $o s p A$ mRNA synthesis following tick-borne murine infection because OspA is downregulated on spirochetes that migrate to the salivary glands of engorged ticks (7), and $\operatorname{sp} A$ mRNA can be detected at 14 but not $30 \mathrm{~d}$ in mice infected with $B$. burgdorferi by syringe challenge (9). In addition, we evaluated flagellin mRNA synthesis, because it is likely that flagellin is expressed throughout the course of infection. As expected, flagellin mRNA could be detected at all the time points tested: 7, 14, 21, and $28 \mathrm{~d}$ suggesting that flagellin is constitutively expressed (Fig. $5 a$ and $b$ ). In these tick-transmission studies, $\operatorname{osp} A$ mRNA was not detected at day 7, but could be detected from day 14 onward. Using the specific primer sets, $o s p E$ mRNA was detected at all the time points that were examined (Fig. $5 a$ and $b$ ). In contrast, $p 21$ mRNA was not identified on day 7 or 14 , and was first detected on day 21 (Fig. $5 a$ and $b$ ). The $o s p E$ and $p 21$ fragments amplified for respective mRNAs on days 14,21, and 28 were sequenced and the identity of $o s p E$ and $p 21$ were confirmed.

Antibodies to P21 can be detected in patients with Lyme disease. Sera from 82 patients with Lyme disease from the Yale Clinic, and sera from 40 patients collected by the CDC were

a
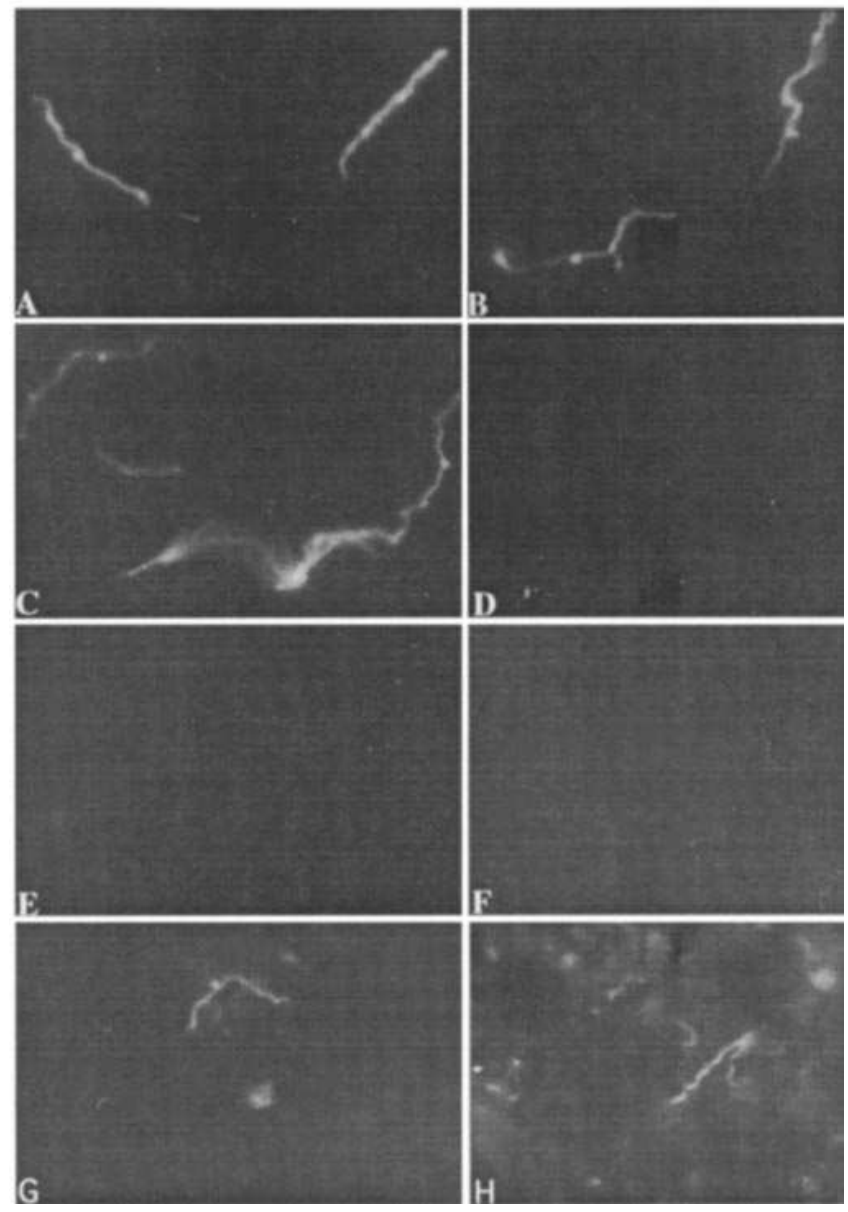

Figure 2. (a) Expression of P21 in B. burgdorferi within ticks. Spirochetes from unfed $(A, C, E, G)$ and fed $(B, D, F, H)$ ticks were fixed with paraformaldehyde and saponin and incubated with flagellin-specific monoclonal antibody (H9724, $[A, B])$, OspA-specific monoclonal antibody (CIII.78, $[C, D]), \mathrm{P} 21$-specific peptide antiserum $(E$, $F)$, or OspE antiserum $(G, H)$. (b) Expression of $\mathrm{P} 21$ by $B$. burgdorferi in infected mice. Representative spirochetes recovered from the peritoneal cavity show rhodamine-immunofluorescence due to binding of anti-B. burgdorferi antibodies $(A, C, E$, and $G)$. Three representative $B$. burgdorferi show FITC-immunofluorescence as a result of the binding of $\mathrm{P} 21$-specific antibodies $(B, D$, and $F)$ and one representative spirochete $(H)$ shows a lack of fluorescence with anti-BSA serum (control). (c) P21-specific antibodies recognize P21 but not OspE. GT (lane 1), GT-P21 (lane 2), and GT-OspE (lane 3) were separated by SDS-PAGE, transferred to nitrocellulose membrane and probed with P21-peptide specific antibodies. $(d)$ P21-specific peptide is recognized by P21: (1) but not by OspE-antisera (2). (e) OspE and P21 antisera, but not P21-specific peptide antiserum, recognize OspE in cultured spirochetes. B. burgdorferi lysates were probed with (1) anti-OspE sera, (2) anti-P21 serum, (3) anti-P21-specific-peptide serum, and (4) anti-BSA serum (control). 

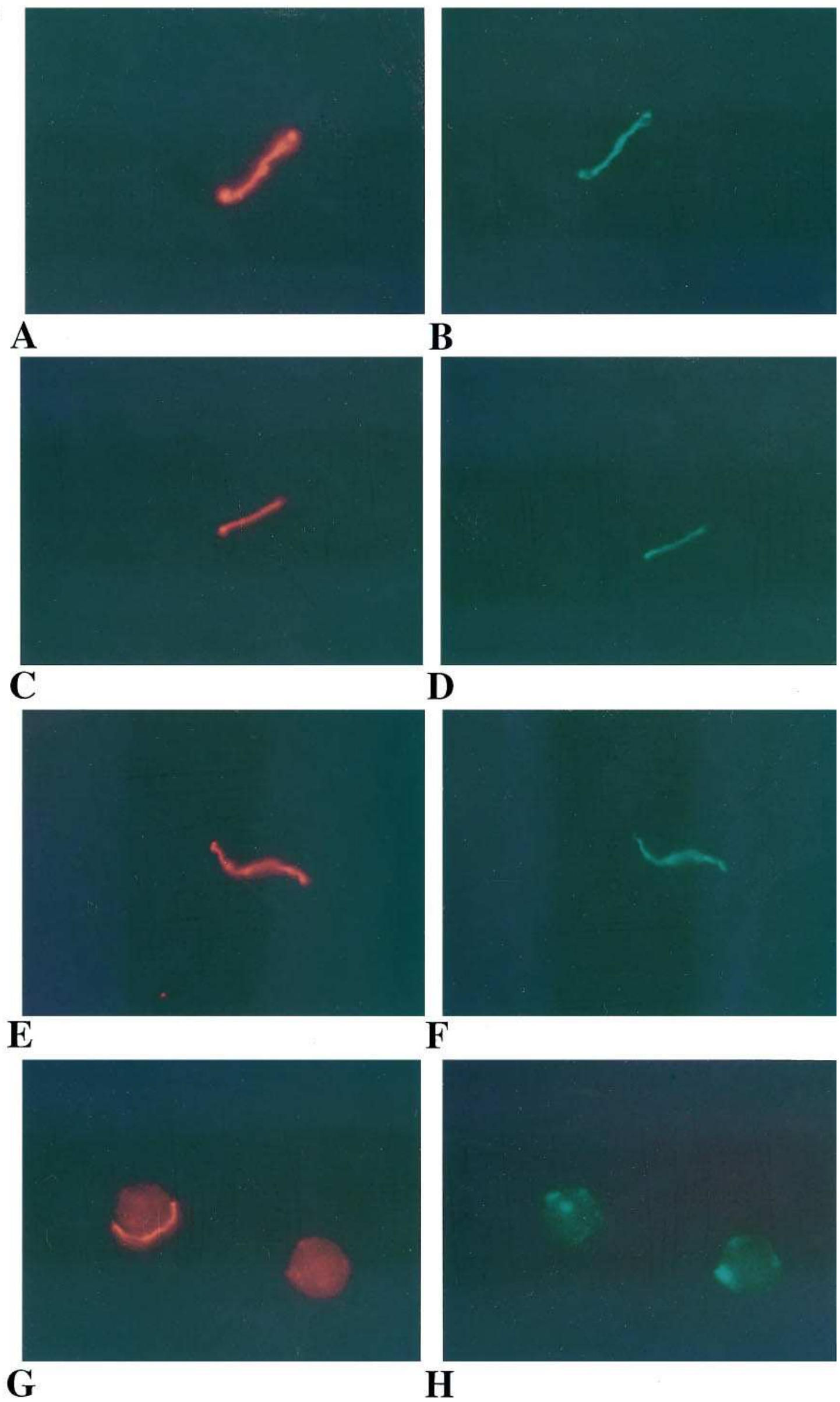

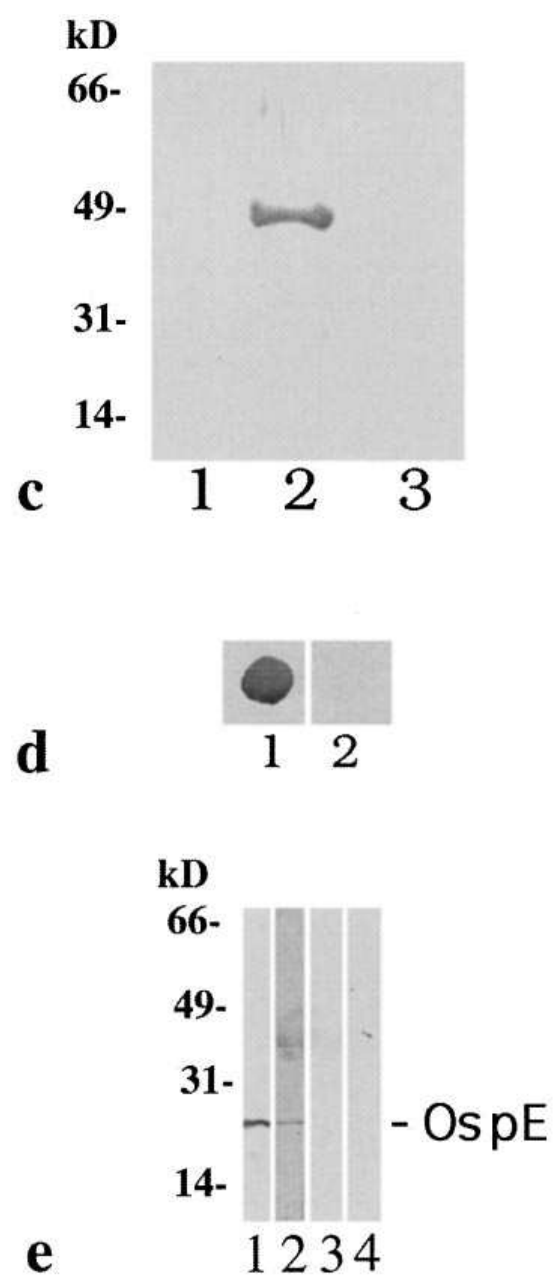

tested for P21 and P21-specific antibodies. P21 antibodies were detected by ELISA and/or immunoblot. As antibodies to OspE may react with $\mathrm{P} 21$, sera that contained antibodies that bound recombinant $\mathrm{P} 21$ were also assessed for reactivity against recombinant OspE and a P21-specific peptide (see Methods). From Yale, $24 \%$ of the patients (20 of 82 ) had antibodies to recombinant P21, all of whom had IgG-specific P21-antibodies. 8 of these 20 patients also had IgM-specific P21-antibodies. Of the 20 patients that had $\mathrm{P} 21$ antibodies by ELISA, 18 sera reacted with P21 in immunoblot. 17 of the 20 P21-positive sera also bound to recombinant OspE. 16 of these 20 sera had antibodies that bound the P21-specific peptide, suggesting that at least some of the antibodies were directed towards P21. A detailed clinical history was available on 56 of the 82 individuals, including 12 of the 20 P21-positive patients. 31 of 56 patients had Lyme disease of greater than 1 mo duration when their sera were collected and 24 individuals had Lyme arthritis. 3 of the 12 P21-positive patients had early-stage disease with erythema migrans and 9 had late-stage disease, including 6 cases of Lyme arthritis. One patient had Lyme carditis which manifested as heart block, and two subjects had neuroborreliosis.

Sera of 40 individuals with well-defined Lyme disease collected by the CDC, 27 (68\%) of which were culture-positive for B. burgdorferi, were also probed for the presence of antibodies to P21, OspE, and a P21-specific peptide by ELISA. 14 of $40(35 \%)$ patients had $\mathrm{IgG}$ and/or IgM antibodies to P21, and 11 had IgG antibodies. 8 of the 14 individuals with P21 antibodies also had antibodies to OspE. 10 patients also had P21specific (P21-pep) antibodies. In general IgM antibodies to $\mathrm{P} 21$ could be detected in patients with Lyme disease $<3$ mo of duration, the vast majority of whom had erythema migrans. IgG antibodies to P21 were detected in 10 patients with a disease course of greater than $1.5 \mathrm{mo}$, and in five of nine $(56 \%)$ of the individuals who had Lyme arthritis. 12 of the 14 P21-positive individuals were also culture-positive for B. burgdorferi. These patients came from diverse areas, including Wisconsin, Michigan, New York, Connecticut, and Massachusetts.

P21 antibodies are not protective. Because certain antibodies that are generated during the first month of murine borreliosis are both protective and disease modulating (18), and P21 is preferentially induced during infection, we determined whether or not P21 antibodies were protective. Six mice immunized with recombinant GT-P21 developed P21 antibodies that could be detected at a titer of at least 1:5000 by immunoblot. The immunized mice were then challenged by attachment of 15 B. burgdorferi-infected I. dammini ticks. Infection (the number of culture-positive mice), and disease (arthritis and carditis) were similar in P21-immunized (six of six mice) and control animals (six of six mice). Moreover, B. burgdorferi infection rates were similar within ticks that engorged upon experimental $(100 \%)$ or control mice $(100 \%)$. Therefore, immunization with $\mathrm{P} 21$ is not protective against tick-borne infection. In addition, the passive transfer of P21-specific antiserum or GT antiserum (control) to mice which were previously inoculated with $10^{4} \mathrm{~B}$. burgdorferi, did not abrogate the infection or modify disease in SCID mice (five of five animals in both groups were culture-positive and had arthritis and carditis). The passive transfer of serum from mice infected with $B$. burgdorferi for $90 \mathrm{~d}$, as expected (18), resulted in spirochete clearance and resolution of active disease (five of five animals were culture-negative for B. burgdorferi and did not have active arthritis or carditis).

\section{Discussion}

B. burgdorferi osp $E$ and $F$ homologues, including $p 21, p G$, $b b k 2.10$, and $b b k 2.11$ are selectively expressed in the infected host; however, nothing is known about the temporal expression of these genes in vivo or the host immunologic responses to these antigens. $o s p E$ and $F$ are on a $45 \mathrm{~kb}$ plasmid, and are expressed both in vitro and in vivo. As the homologues of $o s p E$ and $F$, which share $32-76 \%$ structural similarity $(1,3-6)$, can be located on multiple circular or linear B. burgdorferi plasmids, we determined the location of $p 21$. We found that a full-length $p 21$ probe hybridized at three locations: at $45 \mathrm{~kb}$ and at two other bands which moved more slowly than the $49 \mathrm{~kb}$ plasmid (Fig. $1 \mathrm{a}$ ). The band at $45 \mathrm{~kb}$ represents the plasmid containing $\operatorname{osp} E-F(5)$, which has been shown to be circular (6), and the band at $90-100 \mathrm{~kb}$ region is probably the plasmid containing $b b k 2.10$ (1). A p 21 -specific probe localized $p 21$ to a different plasmid than that of $o s p E$, and two-dimensional electrophoresis of B. burgdorferi DNA suggested the circular nature of this plasmid. These data show that B. burgdorferi can contain several homologues of $\operatorname{ss} E-F$. However, diverse strains may not contain all members of this gene family, and the location of these genes within the genome may differ in se- 
lected isolates. Within B. burgdorferi $\mathrm{N} 40$, ospE and $p 21$ are located on separate plasmids.

The detection of $\operatorname{ssp} E$ and $p 21 \mathrm{mRNA}$ and $\mathrm{OspE}$ and P21 antibodies during murine infection indicates that these genes are sequentially expressed. Antibodies that bound P21 or OspE were first detected $7 \mathrm{~d}$ after infection, and could represent a response directed at either P21 or OspE because of potential cross reactivity. The appearance of $\mathrm{P} 21$-specific antibodies $28 \mathrm{~d}$ after infection suggests that the early response to P21 may be a result of the non-specific binding of OspE antibodies with recombinant $\mathrm{P} 21$, or that the early $\mathrm{P} 21$ response is not directed towards the P21-specific peptide. The former hypothesis is supported by the initial appearance of $p 21$ mRNA in infected animals at day 21 , whereas $o s p E$ mRNA is readily detectable $7 \mathrm{~d}$ after infection (the first time point examined).

Temperature can influence the regulation of $\operatorname{spp} C$ (8) and members of the $\operatorname{ss} E / F$ gene family (20); however, additional factors must influence gene expression because OspC (8), but not $\mathrm{P} 21$, is detectable in the engorged tick. As the regulation of OspA and OspC synthesis predominantly occurs within the engorging ticks $(7,8)$, and our data show that P21 is not synthesized in the flat or engorged tick, $p 21$ appears to be the first example of a gene that is selectively expressed within the mammalian host.

Comparison of flagellin and osp $A$ expression during the early stages of murine infection indicated that $\operatorname{sp} A \mathrm{mRNA}$ synthesis is also temporally regulated. flagellin mRNA was detected at all the time points, indicating that it is constitutively expressed. osp $A$ mRNA, however, was not detected until $14 \mathrm{~d}$ after tick-borne infection and continued to be identified at 21 and $28 \mathrm{~d}$. This is in contrast with previous studies showing that in mice inoculated intradermally with $10^{4}$ spirochetes, osp $A$ mRNA was detected at 14, but not $30 \mathrm{~d}$ (9). The difference at $30 \mathrm{~d}$ may be due to the method of $B$. burgdorferi infection: natural tick-transmission compared with experimental syringe inoculation. OspA is down-regulated by B. burgdorferi in the salivary glands of engorged ticks, whereas OspA is a major antigen of B. burgdorferi in culture $(7,17)$. Thus, it is possible that laboratory-cultured spirochetes express OspA in the initial stages of murine infection, whereas tick-transmitted $B$. burgdorferi do not synthesize OspA immediately, but express the gene later. Appearance of $\operatorname{sp} A$ mRNA, but a lack of OspA antibodies, in the initial phase of murine infection could also suggest that OspA synthesis may be regulated at the level of translation. In addition, mice immunized against OspA and then challenged with spirochetes that have adapted to the host (using infected tissue transplants) are fully susceptible to disseminated infection and disease, in contrast to mice challenged with cultured spirochetes or by tick challenge (10). This further suggests that OspA expression does not occur in hostadapted spirochetes or is expressed by some, but not all, spirochetes.

A P21-specific response can also be detected in patients with Lyme disease. Our group previously reported that $11 \%$ and $15 \%$ of the sera from patients with early- and late-stage Lyme disease contained OspE-specific antibodies (5). This study indicates that $28 \%$ of the patients had OspE and/or P21 antibodies. The differences in the percentage of seropositive patients may be due to the increased sensitivity of the ELISA and the diversity of the current patient population that in-

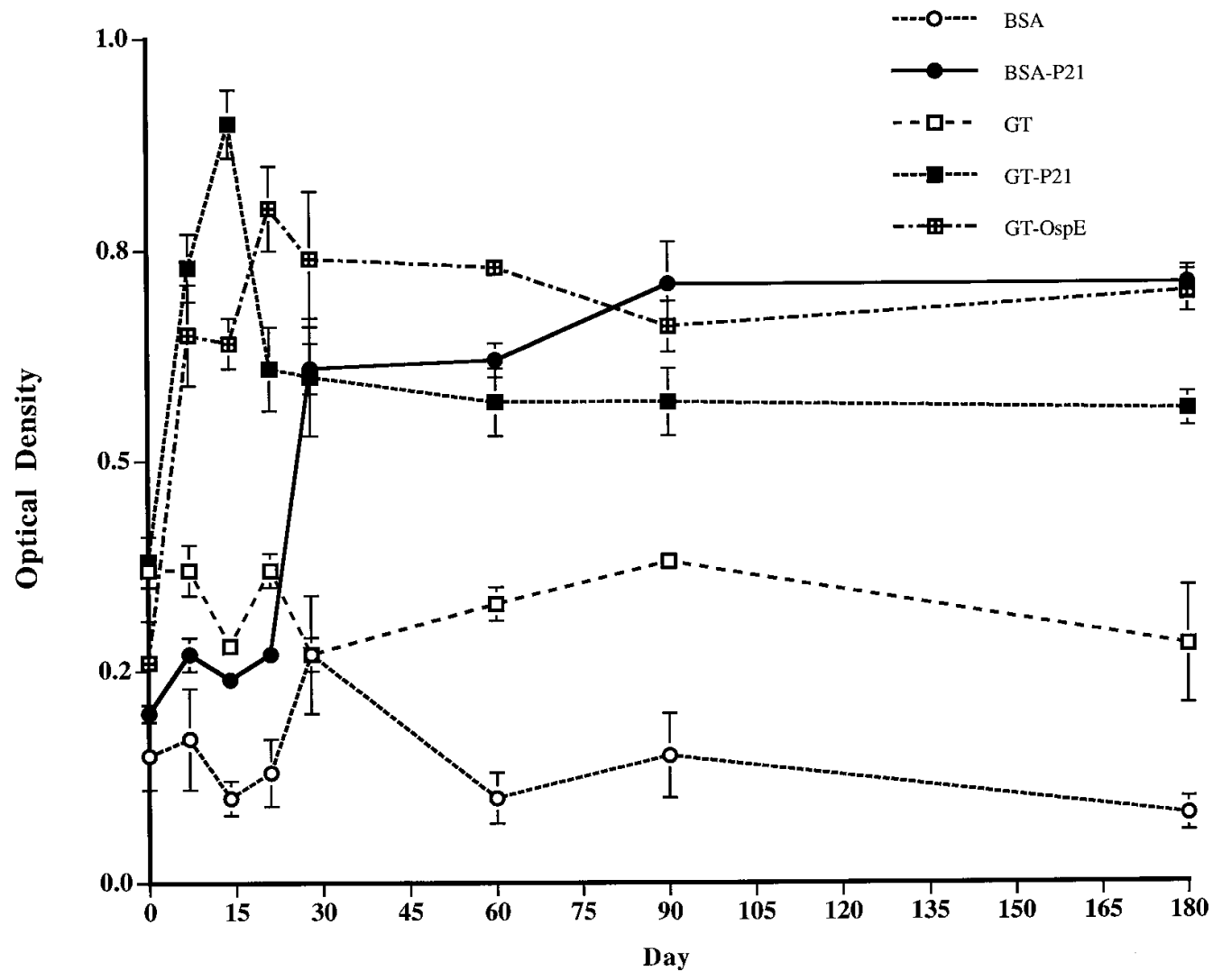

Figure 3. P21-specific IgG titers in B. burgdorferi-infected mice. Animals were infected with $10^{4} B$. burgdorferi and blood was collected on selected days. Each point represents the average and SD of three separate ELISA values of pooled sera from five animals. 
cludes samples from both Yale and CDC. Reactivity of these sera against a 10 amino acid peptide specific to $\mathrm{P} 21$ helped distinguish between OspE and P21 reactivity. 16 of 20 P21-OspE positive sera from Yale had antibodies against the P21-specific peptide. This suggests that $\mathrm{P} 21$-specific antibodies are synthesized during human B. burgdorferi infection, and supports our murine in vivo expression studies. Moreover, it is possible that the other four patients may have developed P21-specific antibodies, but that none of these antibodies were directed towards this P21-specific epitope. In general, an IgM response to P21 was detected within the first several months after infection, and an $\mathrm{IgG}$ response was identified at later time points, suggesting that $p 21$ is expressed in the early stages of human infection, consistent with the data indicating that P21 is synthesized within the first month of murine infection.

Although P21 and members of OspE-F antigen family are induced in the host, $\mathrm{P} 21$-antibodies were only detected in $28 \%$ of the patients. As this gene family is fairly diverse, it is possible that $p 21$ was not present in all the $B$. burgdorferi isolates that infected the patients, and that closely related antigens are not cross-reactive with $\mathrm{P} 21$ or OspE. For example, antibody to Bbk2.10, an OspF homologue, does not cross react with OspF (1). Therefore, it is possible that P21 antibodies are detectable when the infecting $B$. burgdorferi strain contains $P 21$, whereas in other patients, some other homologue (5) of the ospE-F gene family is expressed. Antibodies to P21 were most evident after 1 mo of infection; however, a correlation between a specific manifestation of Lyme disease and the presence of P21antibodies in human sera was not evident.

Immunization with P21 did not protect mice from B. burgdorferi infection or disease, even though immunized mice developed high titers of P21-specific antibodies (levels, which in the case of OspA, have been shown to be protective) (16). This is consistent with lack of expression of $P 21$ in the flat or engorged ticks, or in the initial phase of murine infection, therefore resulting in an inability to elicit protective immunity. Moreover, although OspE and $\mathrm{F}$ antibodies can partially destroy spirochetes within engorged ticks (21), antibodies to recombinant P21 that may cross react with OspE do not serve this function because the spirochete load in ticks that fed on

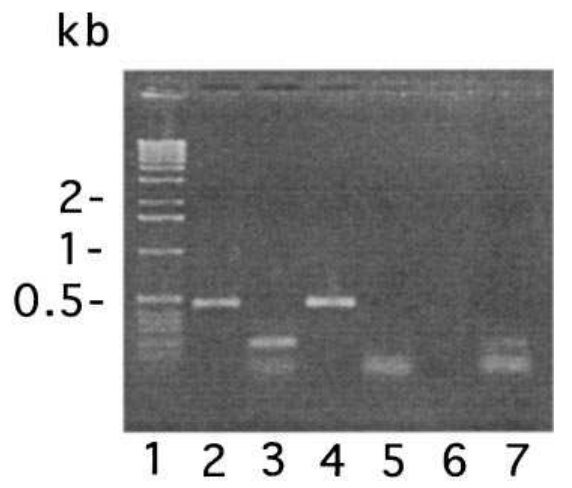

Figure 4. Specificity of the $o s p E$ and $p 21$ primers. Molecular weight marker (lane 1), p21-specific primers with B. burgdorferi DNA as the template (lane 2), ospE-specific primers with B. burgdorferi DNA (lane 3), $p 21$-specific primers with $p 21$ plasmid DNA (lane 4), ospEspecific primers with $p 21$ plasmid DNA (lane 5), $p 21$-specific primers with $o s p E$ plasmid DNA (lane 6), and $o s p E$-specific primers with osp E plasmid DNA (lane 7).
P21-immunized mice was not reduced. Barthold et al. (18) have shown that sera from mice infected with $B$. burgdorferi for $90 \mathrm{~d}$, but not from mice immunized with heat-killed spirochetes, cleared spirochetes from SCID mice that had been infected with $B$. burgdorferi for up to $4 \mathrm{~d}$ and induced arthritis resolution when serum was transferred after arthritis was underway. These data suggest that specific antibodies to antigens selectively expressed in vivo by $B$. burgdorferi are involved in spirochetal clearance and disease modulation. As P21 is an in vivo induced antigen, we also sought to determine whether P21 antibodies had protective or arthritis-modulating capacity in the SCID mouse model system. P21-specific antibodies did not alter infection or disease in the SCID mice.

a

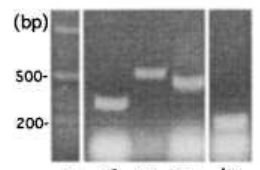

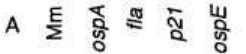

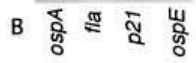
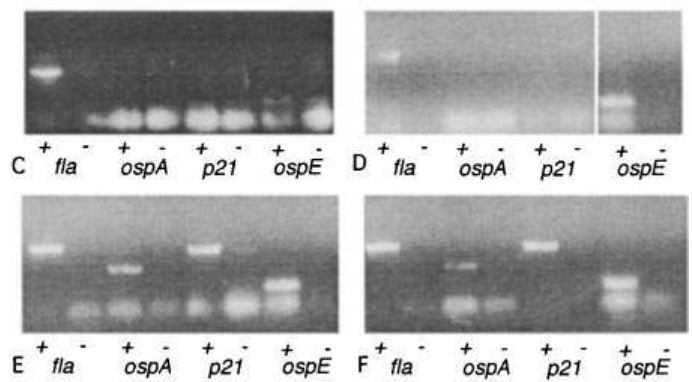

b
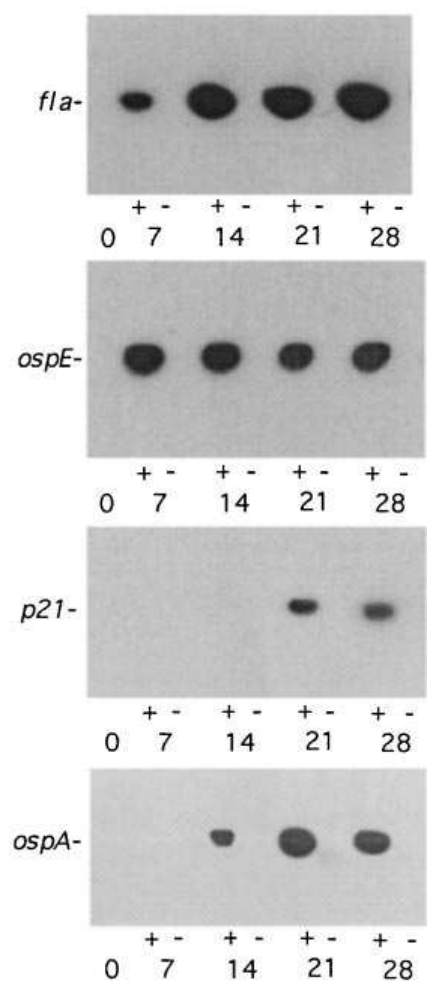

Figure 5. Expression of $p 21$ in infected mice. Total RNA isolated from the spleens of infected mice was used for RTPCR. The cDNA was amplified with specific sets of primers for flagellin (fla), osp A, p21, and $\operatorname{sp} E$. (a) Ethidium bromide-stained RT-PCR products: $(A)$ Sizes of $o s p A$, fla, $p 21$, and $o s p E$ fragments amplified from $B$. burgdorferi DNA. $(B)$ day $0,(C)$ day $7,(D)$ day $14,(E)$ day 21 , and $(F)$ day 28 after $B$. burgdorferi infection. With RT $(+)$, without RT (control, [-]). (b) Southern blot analysis of the RT-PCR products. 
Our data indicate that $p 21$ is synthesized or upregulated in the early stage of infection of the vertebrate host because $p 21$ mRNA and P21 antibodies are detected within a few weeks after infection. In contrast, a closely related gene, ospE, is expressed in both the host and vector, whereas $p 21$ is constitutively expressed several weeks after mammalian infection. Therefore, variation in protein expression by B. burgdorferi may be much more extensive than previously believed and the induction of specific genes may occur at various stages of the spirochetal lifecycle. Differential expression of OspA and OspC occurs in engorged ticks, most likely in response to the stimuli provided, whether direct or indirect, by the incoming host blood meal. Then, as the spirochete enters the mammalian host, P21 synthesis in induced. Therefore, spirochetes in engorged ticks appear to differ from spirochetes in the vertebrate host, and variation in protein expression continues as infection progresses. Knowledge regarding the synthesis of $B$. burgdorferi proteins during its lifecycle should provide a greater understanding of the pathogenesis of Lyme disease.

\section{Acknowledgments}

We gratefully thank Ms. Patty Farrel and Dr. Louise-Marie Dembry for their help with the pulsed-field gel electrophoresis, Ms. Deborah Beck and Ms. Rhonda Bangham for their technical assistance with animal sacrifice and spirochete culture, and Dr. Martin E. Schriefer from the CDC for supplying sera from patients with Lyme disease.

This work was supported by grants from the National Institutes of Health AI-30548, AI-37993, AI-45253, AI-49387, Centers for Disease Control and Prevention (U5O-CCU-106581), Mathers and Arthritis Foundations, and American Heart Association. E. Fikrig is a Pew Scholar.

\section{References}

1. Akins, D.R., S.R. Porcella, T.G. Popova, D. Shevchenko, S.I. Baker, M. Li, M.V. Norgard, and J.D. Radolf. 1995. Evidence for in vivo but not in vitro expression of a Borrelia burgdorferi outer surface protein $\mathrm{F}(\mathrm{OspF})$ homologue. Mol. Microbiol. 18:507-520.

2. Champion, C.I., D.R. Blanco, J.T. Skare, D.A. Haake, M. Giladi, D. Foley, J.N. Miller, and M.A. Lovett. 1994. A 9.0-kilobase-pair circular plasmid of Borrelia burgdorferi encodes an exported protein: evidence for expression only during infection. Infect. Immun. 62:2653-2661.

3. Wallich, R., C. Brenner, M.D. Kramer, and M.M. Simon. 1995. Molecular cloning and immunological characterization of a novel linear-plasmid-encoded gene, $p G$, of Borrelia burgdorferi expressed only in vivo. Infect. Immun. 63:
3327-3335.

4. Suk, K., S. Das, W. Sun, B. Jwang, S.W. Barthold, R.A. Flavell, and E. Fikrig. 1995. Borrelia burgdorferi genes selectively expressed in the infected host. Proc. Natl. Acad. Sci. USA. 92:4269-4273.

5. Lam, T.T., T.P. Nguyen, R.R. Montgomery, F.S. Kantor, E. Fikrig, and R.A. Flavell. 1994. Outer surface proteins E and F of Borrelia burgdorferi, the agent of Lyme disease. Infect. Immun. 62:290-298.

6. Stevenson, B., K. Tilly, and P.A. Rosa. 1996. A family of genes located on four separate 32-kilobase circular plasmids in Borrelia burgdorferi B31. J. Bacteriol. 178:3508-3516.

7. de Silva. A.M., S.R. Telford, L.R. Brunet, S.W. Barthold, and E. Fikrig. 1996. Borrelia burgdorferi OspA is an arthropod-specific transmission-blocking Lyme disease vaccine. J. Exp. Med. 183:271-275.

8. Schwan, T.G., J. Piesman, W.T. Golde, M.C. Dolan, and P.A. Rosa. 1995. Induction of an outer surface protein on Borrelia burgdorferi during tick feeding. Proc. Natl. Acad. Sci. USA. 92:2909-2913.

9. Montgomery, R.R., S.E. Malawista, K.J. Feen, and L.K. Bockenstedt. 1996. Direct demonstration of antigenic substitution of Borrelia burgdorferi ex vivo: exploration of the paradox of the early immune response to outer surface proteins A and C in Lyme disease. J. Exp. Med. 183:261-269.

10. Barthold, S.W., E. Fikrig, L.K. Bockenstedt, and D.H. Persing. 1995. Circumvention of outer surface protein A immunity by host-adapted Borrelia burgdorferi. Infect. Immun. 63:2255-2261.

11. Schutzer, S.E., P.K. Coyle, J.J. Dunn, B.J. Luft, and M. Brunner. 1994 Early and specific antibody response to OspA in Lyme disease. J. Clin. Invest. 94:454-457.

12. Kalish, R.A., J.M. Leong, and A.C. Steere. 1995. Early and late antibody responses to full-length and truncated constructs of outer surface protein A of Borrelia burgdorferi in Lyme disease. Infect. Immun. 63:2228-2235.

13. Ferdows, M.S., and A.G. Barbour. 1989. Megabase-sized linear DNA in the bacterium Borrelia burgdorferi, the Lyme disease agent. Proc. Natl. Acad. Sci. USA. 86:5969-5973.

14. Das, S., D. Shraga, C. Gannon, T.T. Lam, S. Feng, L.R. Brunet, S.R Telford, S.W. Barthold, R.A. Flavell, and E. Fikrig. 1996. Characterization of a 30-kDa Borrelia burgdorferi substrate-binding protein homologue. Res. Microbiol. 147:739-751.

15. Sears, J.E., E. Fikrig, T.Y. Nakagawa, K. Deponte, N. Marcantonio, F.S Kantor, and R.A. Flavell, 1991. Molecular mapping of Osp-A mediated immunity against Borrelia burgdorferi, the agent of Lyme disease. J. Immunol. 147: 1995-2000.

16. Fikrig, E., S.W. Barthold, F.S. Kantor, and R.A. Flavell. 1990. Protection of mice against the Lyme disease agent by immunizing with recombinan OspA. Science (Wash. DC). 250:553-556.

17. Fikrig, E., E.D. Huguenel, R. Berland, D.W. Rahn, J.A. Hardin, and R.A. Flavell. 1992. Serologic diagnosis of Lyme disease using recombinant outer surface proteins A and B and flagellin. J. Infect. Dis. 165:1127-1132.

18. Barthold, S.W., M. deSouza, and S. Feng. 1996. Serum-mediated resolution of Lyme arthritis in mice. Lab. Invest. 74:57-67.

19. Sadziene, A., B. Wilske, M.S. Ferdows, and A.G. Barbour. 1993. The cryptic ospC gene of Borrelia burgdorferi B31 is located on a circular plasmid. Infect. Immun. 61:2192-2195.

20. Stevenson, G., T.G. Schwan, and P.A. Rosa. 1995. Temperature-related differential expression of antigens in the Lyme disease spirochete, Borrelia burgdorferi. Infect. Immun. 63:4535-4539.

21. Nguyen, T.P., T.T. Lam, S.W. Barthold, S.R. Telford, R.A. Flavell, and E. Fikrig. 1994. Partial destruction of Borrelia burgdorferi within ticks that engorged on OspE- or OspF-immunized mice. Infect. Immun. 62:2079-2084. 